\title{
Light Red Shift in Cosmic Background Photon Gas
}

\author{
Jikang Chen \\ College of Physics, Nanjing Normal University, Nanjing, China \\ Email: chenjikang@njnu.edu.cn
}

Received 8 October 2014

\begin{abstract}
There is a stable cosmic background photon gas fulfilled in our universe and a very weak damping force is acting on every traveling photon, then the light red shift has been observed at a large distance. When a photon travels in the cosmic background photon gas, its frequency continually lowers and at last diminishes in the photon gas as a low velocity one. The decay factor of traveling photons has been estimated; the light red shift is nonlinear in space, and our visual field is finite although the universe is infinite.
\end{abstract}

Keywords

Photon, Damping, Red Shift, Nonlinear

\section{Introduction}

Spectrographic analysis of light received from distant galaxies shows that certain prominent spectral lines identified in spectroscopic studies in the laboratory are shifted very significantly toward the lower frequency end of the visible spectrum. This red shift may be interpreted as a Doppler shift arising from the velocity of recession of the source. It is also known that the velocities calculated from these Doppler shifts are directly proportional to the distances of the source from us determined by independent means. But the red shift can be caused by the damping effect of the photon gas at cosmic background temperature in space.

\section{The Rest Mass and Magnetic Momentum of a Photon}

Every photon should have same rest (stand by) mass $m_{\mathrm{s}}$ and same rest frequency $v_{s}$. The rest mass of a photon has been estimated based on the cosmic background temperature $\left(T_{\mathrm{CB}}=2.725 \mathrm{~K}\right)$ by the method of dealing with an open state of thermal equilibrium [1],

$$
m_{s}=7.725(3) \times 10^{-40} \mathrm{~kg},
$$

and the rest frequency of a photon is

$$
h v_{s}=m_{s} c^{2}, \quad v_{s}=1.0479(4) \times 10^{11} \mathrm{~Hz} .
$$

The ratio between a traveling photon's frequency and its rest frequency is just one relativistic factor, 


$$
v=\gamma v_{s},
$$

and the group velocity of the traveling photon is

$$
c_{g}^{2}=c^{2}\left(1-\gamma^{-2}\right) .
$$

Considering the Faraday rotation effects, it is reasonable to suppose that every photon has the same proper magnetic moment and it has been estimated [1]:

$$
m_{p}^{\prime 2}=\frac{9 h c^{3}}{20 \pi v_{s}^{2} \mu_{0}}, \quad m_{p}^{\prime}=4.305(2) \times 10^{-13} \mathrm{Am}^{2} .
$$

\section{The Photon Gas Is Fulfilled Our Universe}

In the thermal radiation of a black body, the intensity is composed of two parts: one is the wave noise, and the other is the shot noise. So the Planck formula can be separated mathematically into two parts: the wave noise is in the form of electromagnetic wave [1],

$$
I_{w}(f)=\frac{2 \pi c^{-2} h f^{3} \exp (-h f / k T)}{\exp (h f / k T)-1},
$$

and the shot noise is in the form of traveling photons,

$$
I_{p}(v)=2 \pi c^{-2} h v^{3} \exp (-h v / k T) .
$$

Then the number density of the background photon gas at high temperature is

$$
n(v)=8 \pi c^{-3} v^{2} \exp (-h v / k T), \quad T \gg T_{\mathrm{CB}},
$$

at general temperature the number density of the background photons is increased by a factor [1]:

$$
n(v)=8 \pi c^{-3} C_{T} v^{2} \exp (-h v / k T) .
$$

There is a stable cosmic background photon gas fulfilled in our universe. The magnetic property of photon's rest energy will cause negative mechanical pressure in the photon gas, and the photon's kinetic energy will cause the positive one. The total mechanical pressure should be zero at the cosmic background temperature.

\section{The Damping of Traveling Photons in Cosmic Background Photon Gas}

In the proper system of cosmic background photon gas, the visual transverse characteristic magnetic moment of a transverse polarized traveling photon is reduced by two relativistic factors from the proper magnetic moment of the photon [1].

$$
m_{p, T}=\gamma^{-2} m_{p}^{\prime},
$$

and the visual transverse characteristic electric moment of the photon is at the same order [1].

$$
p_{e, T}=c_{g}^{-1} \gamma^{-2} m_{p}^{\prime} .
$$

The interaction between the traveling photon and the cosmic background photon gas is essentially magnetic and electronic, so the average proper damping force is reduced by two relativistic factors of the traveling photon,

$$
f_{d}^{\prime} \propto-v^{-2},
$$

When a photon travels in the cosmic background photon gas, its frequency continually lowered and at last it is diminished in the photon gas as a low velocity one. The frequency decay of a traveling photon is

$$
\frac{\mathrm{d} v}{\mathrm{~d} t}=-C v^{-2} .
$$

The general solution is

$$
v_{2}^{3}-v_{1}^{3}=-D_{p}\left(t_{2}-t_{1}\right),
$$


Table 1. The largest distance of a traveling photon can reached.

\begin{tabular}{cccc}
\hline Light & Frequency & Wave length & $L_{\max }$ (light year) \\
\hline Infrared & $3.00 \times 10^{14} \mathrm{~Hz}$ & $1000 \mathrm{~nm}$ & $3.0 \times 10^{8}$ \\
Red & $4.61 \times 10^{14} \mathrm{~Hz}$ & $650 \mathrm{~nm}$ & $1.1 \times 10^{9}$ \\
Blue & $6.67 \times 10^{14} \mathrm{~Hz}$ & $450 \mathrm{~nm}$ & $3.3 \times 10^{9}$ \\
NUV & $7.50 \times 10^{14} \mathrm{~Hz}$ & $400 \mathrm{~nm}$ & $4.7 \times 10^{9}$ \\
LUV & $3.00 \times 10^{15} \mathrm{~Hz}$ & $100 \mathrm{~nm}$ & $3.0 \times 10^{11}$ \\
SUV & $3.00 \times 10^{16} \mathrm{~Hz}$ & $10.0 \mathrm{~nm}$ & $3.0 \times 10^{14}$ \\
Soft-X & $3.00 \times 10^{17} \mathrm{~Hz}$ & $1.00 \mathrm{~nm}$ & $3.0 \times 10^{17}$ \\
Hard-X & $3.00 \times 10^{18} \mathrm{~Hz}$ & $1.00 \times 10^{-1} \mathrm{~nm}$ & $3.0 \times 10^{20}$ \\
Super Hard X & $3.00 \times 10^{19} \mathrm{~Hz}$ & $1.00 \times 10^{-2} \mathrm{~nm}$ & $3.0 \times 10^{23}$ \\
\hline
\end{tabular}

where $D_{p}$ is the decay factor of traveling photons. The distance of Virgo Cluster is about 59 million light years away [2]. If the observed photon frequency is the pair of easily recognizable absorption lines in the spectrum of potassium, the red shift is between $7.5899 \times 10^{14} \mathrm{~Hz}$ and $7.5593 \times 10^{14} \mathrm{~Hz}$, then

$$
D_{p}=8.9 \times 10^{34} \mathrm{~Hz}^{3} \mathrm{yr}^{-1} .
$$

It is clear that the frequency decay of a photon traveling in the cosmic background photon gas is nonlinear with respect to distance, and the largest distance that a traveling photon can reached in space is limited by its original frequency:

$$
L_{\max }=D_{p}^{-1} v^{3} \text { (light year). }
$$

Table 1 shows some typical light from Infrared to Super Hard X ray, their frequency or wave length, and the largest distance it can reached at the cosmic background temperature in space.

\section{Discussion}

All celestial bodies are moving around and it may be right to think that the recession velocity is directly proportional to the distances, but it is difficult to understand that the recession is comparable with light speed for so large and so many stars. It is clear that the light red shift is the nonlinear decay of photons; the light maximum transmission distance is limited by its cubic frequency; our visual field is finite although the universe is infinite; and some stars beyond our visual field as black hole can be determined by gravitational force or electromagnetic wave of lower frequency.

\section{References}

[1] Chen, J.K. (2010) The Relativistic Dynamics of Photon Gas. http://arxiv.org/ftp/arxiv/papers/1003/1003.0255.pdf

[2] Kittel, C. (1973) Mechanics. 2nd Edition, McGraw-Hill, Inc., 318, 319. 